MATEC Web of Conferences 40, 02021 (2016)

DOI: $10.1051 /$ matecconf/20164002021

(C) Owned by the authors, published by EDP Sciences, 2016

\title{
The Application of Simulation Technology in the Parameter Optimization of Casting Process
}

\author{
Wenwei Zhuang ${ }^{1}$, Zhixin Chen ${ }^{1}$, Sufen Yan ${ }^{2}$, Haiying Zhou ${ }^{1}$ \\ 1. School of Mechanical and Electronic Engineering, East China university of Technology, Nanchang, Jiangxi, China \\ ${ }^{2}$ Jiangxi Institute of Computing Technology, Nanchang, Jiangxi, China
}

\begin{abstract}
Since the casting process of large castings is complicated, the optimization of process parameters has a significant impact on product quality. In this paper, main technical parameters of male thread casting in screw expansion power machine were compared and analyzed with simulation analysis method and an applicable speed and temperature for male thread casting was obtained by mainly considering the entrapped air and the surface defect density. In general, the analysis methods provided herein, offers a viable way to optimize the quality of large machine components while casting. This can benefit the productivity and cost saving.
\end{abstract}

\section{Introduction}

The filling process during casting is unstable and hard to control. It accompanied by liquid metal flow, temperature variation, flow area shifting and other complex phenomenon. It can cause product defects, such as blowholes, short-poured and cold shut. The casting process can be optimized and the quality of castings can be improved, if we conduct an analog computation to prognosis the potential defects during the filling process. The research on numerical simulation for filling process, which based on temperature field simulation technology, sprung up since 1980s. At first, the researchers simulated the filling process of liquid metal flow into a rectangular cavity with two-dimensional method, and it proved to be a stepwise vertical filling process. On this basis, the computer numerical simulation for filling process started blooming. The theory of simulation analysis study on filling process, which oriented to the accuracy and practicably, has been consummating. Commonly used algorithms are SOLA-VOF, SIMPLER, MAC, SMAC, COMMIX (Among these, the most practical algorithm is SOLA-VOF. Lots of improving schemes are aim at it.). These algorithms contain the momentum equation, continuity equation, energy equation, the volume function equation and turbulent kinetic energy equation, and so on. At present, the filling process simulation has been developed to the three-dimensional, which means more factors are considered during the simulation and the results are more reliable. With the rapid development of computers, we need less time to calculate and the value of simulation has been enhanced. As a result, simulation analysis has become a necessary process before actual production.
So far, the research and application of numerical simulation for filling and solidification process has move from sand casting into metal casting. It's a progress we make in simulation technology.

\section{Process Parameter}

There are various of process parameters that could affect the quality of foundries, such as pressure parameters (injection pressure, injection pressure); speed parameters (speed runner, injection velocity); temperature parameters (liquid metal temperature, mold temperature); time parameters (filling time, holding pressure time, leaving die time); the ply of oddments and the dosage of alloy liquid. The vital questions we should concern about during the casting production are: How to select the appropriate parameters? How to arrange a reasonable match for parameters in order to get the best results. As the parameters are too much to be discussed, this article mainly shows how does temperature and speed affect quality.

\subsection{The Influence of temperature}

Casting temperature should ensure the flux an excellent fluidity during the injection process. The casting temperature should be determined depending on the transfer distance, the cool condition of foundries, the specifications of alloys, rate of flow and other factors. Generally, the casting temperature should be $50 \sim 110^{\circ} \mathrm{C}$ higher than the alloy liquids temperature.

Casting temperature has a great influence on the microstructure, mechanical properties, crack orientation, and exterior quality. Microstructure: Raise the casting temperature can increase the tendency of the ingot grain. 
If the temperature was raised within a certain range, the ingot will have a deeper liquid pool; the temperature gradient of the front of crystallization becomes steep. Meanwhile, the cooling rate appeared faster during crystallization and the structure inside the crystal was refined, in the meantime, the tendency of forming into columnar crystals or feather crystal increased.

Also, raise casting temperature can minimize the size of the dangling crystal in the liquid pool, which could lower the tendency of forming a crystalline compound, and improve the exhaust-feeding conditions and the density is improved. Otherwise, the viscosity of the melt will increase, feeding conditions will be deteriorated, and defect the oxide film.

Mechanical Property Raise the temperature within a certain range may improve the mechanical properties of hard-cast alloy ingot. However, the mechanical properties of soft cast alloy ingot will decrease because of the grain size. Whether it's hard or soft alloy ingot, the difference between longitudinal and transverse mechanical properties is quite large. Bring down the casting temperature may reduce mechanical properties of crystalline order.

Ceteris paribus, while increasing the casting temperature, liquid hole became deeper, the tendency of forming into columnar grain increased. Furthermore, it can increase the thermal brittleness of alloy and the crack tendency. In addition, with the solidified shell wall of ingot thinning, some defects like pull marks, cracks or leak segregation could appear under such static pressure, and reduce the tendency of turning into cold traps.

\subsection{The Influence of Speed}

As the temperature, speed is one of the factors that have significant impact on the quality of castings. During the filling process, an improper speed can cause irrational sequential while liquid metal filling the cavity and unstable or overtime filling process. All above can result in casting defects. If the speed or flow is inadequate, there can be a cold shut or short-pouring. On the contrary, overranging speed leads to jet phenomenon. There may emerge inclusion or iron beans. Moreover, excessive velocity impact mold can damage the model wall; bring defects like blowholes or meat trachoma. Filling process also affects the temperature field of the casting. Speed and temperature has mutual effect on each other, both of them can influence the quality of castings.

Numerical simulation of the flow process (flow field) and heat transfer (temperature field) became core of casting forming simulation.

\section{Simulation Technology}

In the filling process of casting, the motion of fluid is dominated by the physical conservation law. Basic laws of conservation include: conservation of mass, conservation of momentum and conservation of energy. The governing equation is a mathematical description of these conservation laws.
Eq. (1) is conservation of mass, which also known as the continuity equation of fluid motion.

$$
\frac{\partial \rho}{\partial t}+\frac{\partial(\rho u)}{\partial x}+\frac{\partial(\rho v)}{\partial y}+\frac{\partial(\rho w)}{\partial z}=0
$$

Momentum conservation law is a basic law which any flow system must follow. It can be describe as the rate of change of fluid momentum in a unit according to time variation is equal to the sum of various external forces acting on the cell. This is actually in accordance with Newton's second law--the alteration of motion is ever proportional to the motive force impressed. As shown in Eq. (2) :

$$
F=\frac{\partial(m u)}{\partial t}
$$

The conservation of energy is a basic law which the flow system of heat exchange must obey, which described as the rate of increase of energy in a unit equals to the force caused by net heat flow and surface force impressed. Take three-dimensional simulation as an example, the energy equation shown as Eq.(3):

$$
\frac{\partial T}{\partial t}+u \frac{\partial T}{\partial x}+v \frac{\partial T}{\partial y}+w \frac{\partial T}{\partial z}=\frac{\lambda}{c \rho}\left(\frac{\partial^{2} T}{\partial x^{2}}+\frac{\partial^{2} T}{\partial y^{2}}+\frac{\partial^{2} T}{\partial z^{2}}\right)
$$

FLOW-3D, analytic software applied for threedimensional engineering fluid mechanics which this paper use, is suitable for simulation of liquid metal flow and solidification process.

FLOW-3D, officially launched in 1985, is developed by an internationally renowned master hydrodynamics Dr. CW Hirt. It earned a good reputation for its precise calculation result and accurate simulation of practical problem and calculating skills of CFD (Computational Fluid Dynamics). Its special technique--FAVOR, provides more accurate and efficient solutions for complicated issues focused on free surface, as to metal casting. It has three features:

\section{(1) Multi-block Grid}

It needs to create a square grid before starting the simulation analysis. As for complicate-shaped, heterogeneous-thicknessed castings, a square with numerous grids is needed for accuracy. For example, it needs to use fine gridding to make the sprue of a casting because it's quite thin. We can divide a casting into parts and change size of grid according to necessary accuracy on each part.

\section{(2) FAVOR Grid-Generation Method}

The traditional FDM, which use structural grid to build geometric model of castings, can shape a casting into staircase and result in computational mistake. Nevertheless, grid subdivision technology of Flow3D, which enable us to split the grid and build complicated model, can help us get an accurate result. 


\section{(3) Tracking Solution for Free Surface of Fluid}

Flow3D is the herald of the calculation method of free surface of fluid (Volume of Fluid). Its unique calculus-TruVOF can track the splashing and aggregation on the front of liquid metal during pressure casting more accurate than ordinary calculus.

\section{Simulation Process and Result}

A screw expanding power generator is a kind of lowgrade thermal energy machines which has a wide application future. The production and processing of key part - screw is vital. To improve products quality, this paper uses the advanced simulation analysis to state the production and male screws processing, the principle of simulation analysis and the use of related software in detail with taking a case of male screw processing. It optimizes the design of male screw processing (mainly riser casting choice), and gets a better one. This paper offers a corresponding solution for this kind of process problems.

Due to the size of male screw is larger, practicality experiments of the equal sizes are impossible. And experiments of small pieces sometimes differ from practical production process too much. At the same time, physical experiments usually only give a result of a certain stage in the manufacturing process, not a complete picture of the entire manufacturing process. There are some limitations. However, the numerical simulation technology on casting process can change production factors in filling and solidification processes into the counting factor which is made by computer simulation. It can reflect the filling and solidification processes of casting and predict accurately positions where defects appear, their sizes and so on in castings by visualization methods. Owing to these specificities of production of large size parts, the use of advanced numerical simulation technique in process has significant meaning that change the recent situation which is only decided by experience in process.

To use Pro/E to draw the screw, to add the related gates and risers, to save it as STL format and then to enter Flow-3D for analysis (as shown in Fig. 1). This case mainly analyzes optimization selection of casting process parameter risers. Therefore, under the premise of the same conditions, I chose different pouring speed and temperature. Then I analyzed and compared entrapped air and surface defect density. And finally I determined the best pouring speed and temperature. The speeds are $1 \mathrm{~m} / \mathrm{s}$ and $2.3 \mathrm{~m} / \mathrm{s}$ as the temperature are $973 \mathrm{~K}$ and $1173 \mathrm{~K}$.

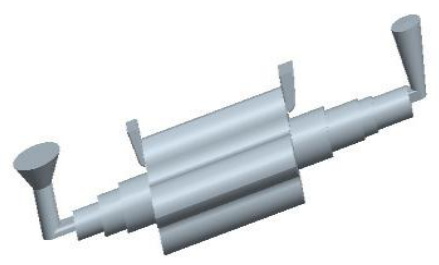

Figure 1. Part model (with gate and riser)

\subsection{The Effect of Speed}

Due to the riser design is better that is fully exhaust, the speed of $1 \mathrm{~m} / \mathrm{s}$ and $2.3 \mathrm{~m} / \mathrm{s}$ have little effect on entrapped air, as Fig. 2 and Fig. 3 showing. But speed has more effect on surface defect density. The more speed it is, the shorter pouring time it takes. And the cooling time is also short. So the temperature of casting-body is uniform. The surface quality is good while surface defect is little, as Fig. 4 and 5 showing. Thus, when the speed is $1 \mathrm{~m} / \mathrm{s}$, the effect is good.

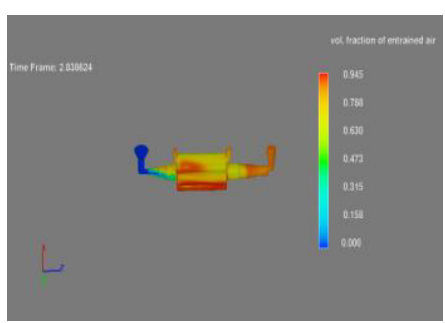

Figure 2. Entrapped air picture speed $1 \mathrm{~m} / \mathrm{s}$, temperature $973 \mathrm{~K}$

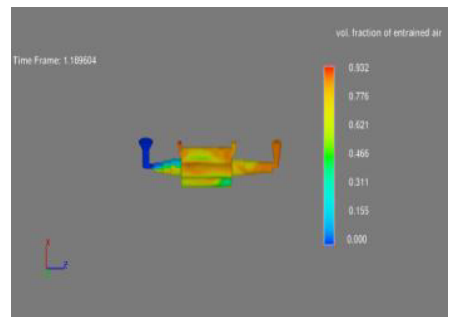

Figure 3. Entrapped air picture speed $2.3 \mathrm{~m} / \mathrm{s}$, temperature $973 \mathrm{~K}$

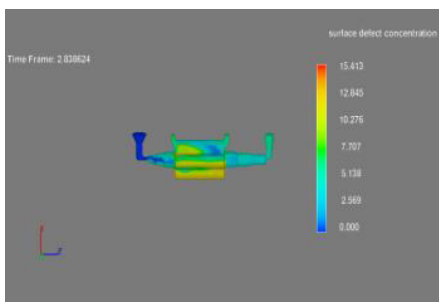

Figure 4. Surface defect density picture speed $1 \mathrm{~m} / \mathrm{s}$, temperature $973 \mathrm{~K}$

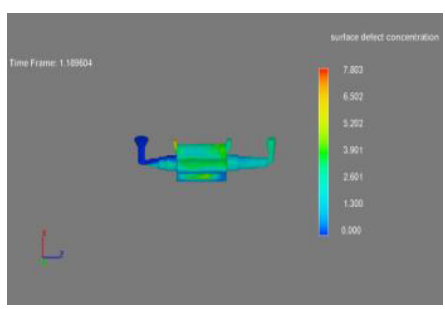

Figure 5. Surface defect density picture speed $2.3 \mathrm{~m} / \mathrm{s}$, temperature $973 \mathrm{~K}$ 


\subsection{The Effect of Temperature}

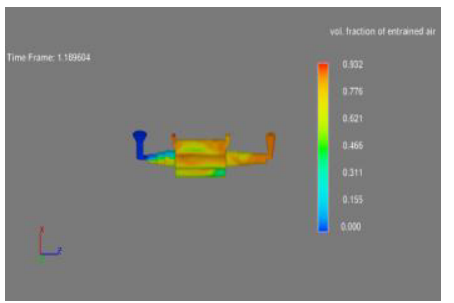

Figure 6. Entrapped air picture speed $2.3 \mathrm{~m} / \mathrm{s}$, temperature $973 \mathrm{~K}$

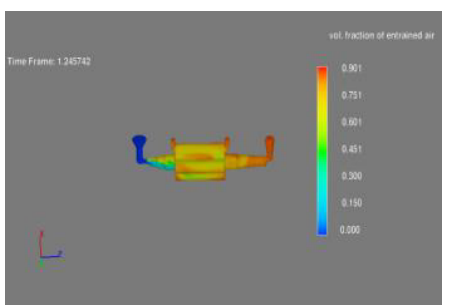

Figure 7. Entrapped air picture speed $2.3 \mathrm{~m} / \mathrm{s}$, temperature $1173 \mathrm{k}$

Fig.6 and 7 shows that entrapped air was hardly affected on account of well-designed riser and fully gas exhausting when the temperature was $973 \mathrm{k}$ and $1173 \mathrm{k}$.

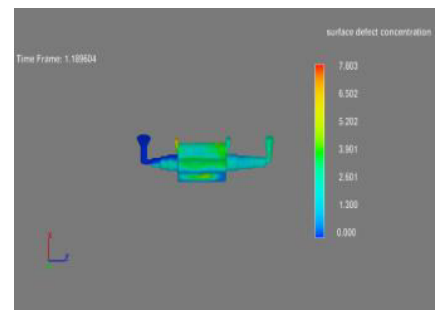

Figure 8. Surface defect density picture speed $2.3 \mathrm{~m} / \mathrm{s}$, temperature $973 \mathrm{~K}$

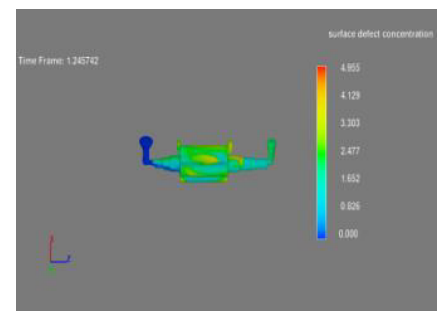

Figure 9. Surface defect density picture speed $2.3 \mathrm{~m} / \mathrm{s}$, temperature $1173 \mathrm{~K}$

Surface defect density was heavily influenced by temperature varying in a certain range. With high quality of metallic surface and good exhaust condition, the density of surface defect is smaller when the temperature was high and metallic flow was unimpeded.

As it shown in Fig. 8 and 9, the defect of surface appears smaller when the temperature was $1173 \mathrm{k}$.

\section{Acknowledgments}

The authors acknowledge the support of science and technology project of Nanchang Municipal Science and Technology Bureau (analog simulation of outline generation and optimum value of blank finishing allowance of screw assembly --- the key components of screw expansion generators). And the support of science and technology project of Science and Technology Department of Jiangxi Province (grant Number 20133BBE50013)

\section{References}

1. Tao Pan, Liao Hencheng, Cheng Jin, Sun Guoxiong. 2005, "The Development of Numerical Simluation in Cast Filling". Process. Casting, Vol.54, No.3:257-260.

2. Sun Ruixia, Liu Qiping, He Bingfeng, Wang Kuangfei, Mi Guofa, 2009, “The Application of Computer Simulation in Optimizing Large Steel-ingot Process". Technology of Metal Casting and Forge Welding, Vol.38, No. 21:5659.

3. Johann Buß, April 2002, GGU-SS FLOWER3D Manual, Braunschweig.

4. Qian Yijun, Cheng Zhaohu, Yu Hao. 2012. "The Application Process of Numerical Simulation in Casting". Precise Forming Engineering. No.07:39-43. 